Dom. Cien., ISSN: 2477-8818

Vol. 4, núm. 1, enero, 2018, pp. 373-384

\title{
Cultura organizacional de servicio al cliente en las entidades públicas del sector educativo
}

\section{Organizational culture of customer service in public entities of the education sector}

\section{Cultura organizacional de atendimento ao cliente em entidades públicas do setor educacional}

\author{
Mayra M. Marcillo-Indacochea ${ }^{\mathrm{I}}$ \\ maymar811@hotmail.com \\ Diana C. Barcia-Pincay II \\ blancaindacochea@hotmail.com \\ Sara G. Soledispa-Reyes ${ }^{\mathrm{III}}$ \\ sarita_1978@outlook.com \\ Jorge I. Calle-García IV \\ narciso.yoza@unesum.edu.ec \\ Blanca Soledad Indacochea-Ganchozo ${ }^{\mathrm{V}}$ \\ blancaindacochea@hotmail.com
}

Recibido: 19 de agosto de 2017 * Corregido: 20 de octubre de 2017 * Aceptado: 15 de noviembre de 2017

I. Magister en Gerencia Educativa, Economista, Universidad Estatal del Sur de Manabí, Jipijapa, Manabí, Ecuador.

II. Tecnóloga en Agroindustrias, Unidad Educativa Fiscal Quince de Octubre, Jipijapa, Manabí, Ecuador.

III. Magister en Contabilidad y Auditoría, Ingeniera Comercial, Universidad Estatal del Sur de Manabí, Jipijapa, Manabí, Ecuador.

Iv. Magister en Desarrollo Educativo, Doctor en Ciencias de la Educación Especialización Educación Primaria, Doctor en Ciencias Pedagógicas, Especialista Superior en Derecho Procesal, Licenciado en Ciencias de la Educación Especialización Educación Primaria, Licenciado en Ciencias Políticas y Sociales, Abogado de los Juzgados y Tribunales de la República, Universidad Estatal del Sur de Manabí, Jipijapa, Manabí, Ecuador.

v. Magister en Agroecología y Agricultura Sostenible, Magister en Administración de Empresas Mención Dirección Financiera, Master en Gestión Ambiental, Doctor en Ciencias Forestales, Diplomado en Autoevaluación y Acreditación Universitaria, Ingeniero Forestal, Universidad Estatal del Sur de Manabí, Jipijapa, Manabí, Ecuador. 


\section{Resumen}

Los cambios están conformando un nuevo tipo de cultura organizacional, caracterizada por una nueva manera de pensar, de visualizar la organización, de desarrollar las actividades. Las instituciones públicas del sector educativo están comprometidas con el éxito es por ello que deben contar con una cultura organizacional enfocada hacia el cliente. Este trabajo tiene como objetivo abordar, desde una perspectiva teórica, la cultura organizacional enfocada al cliente en las instituciones públicas del sector educacional. Para ello se hace una búsqueda de bibliografía mediante diferentes buscadores de Internet, se establecen las palabras claves y se construyen disímiles estrategias de búsqueda. Se seleccionan los documentos pertinentes y se analizan, se extraen y sintetizan determinados contenidos. Se organizan los aspectos de lo general a lo particular y se llegan a conclusiones necesarias a través de la inducción - deducción.

Palabras clave: cultura organizacional; servicio al cliente; instituciones públicas; sector educacional.

\section{Abstract}

The changes are shaping a new type of organizational culture, characterized by a new way of thinking, of visualizing the organization, of developing activities. Public institutions in the education sector are committed to success, which is why they must have an organizational culture focused on the client. The objective of this work is to approach, from a theoretical perspective, the organizational culture focused on the client in the public institutions of the educational sector. To do this, a literature search is done through different Internet search engines, the key words are established and different search strategies are built. The relevant documents are selected and certain contents are analyzed, extracted and synthesized. The aspects of the general to the particular are organized and necessary conclusions are reached through the induction - deduction.

Keywords: organizational culture; customer service; public institutions; educational sector.

\section{Resumo}

As mudanças estão moldando um novo tipo de cultura organizacional, caracterizada por uma nova maneira de pensar, visualizar a organização, desenvolver atividades. As instituições públicas do setor educacional estão comprometidas com o sucesso, e é por isso que eles devem ter uma cultura organizacional focada no cliente. O objetivo deste trabalho é abordar, do ponto de vista teórico, a cultura organizacional focada no cliente nas instituições públicas do setor educacional. Para fazer isso, 
Cultura organizacional de servicio al cliente en las entidades públicas del sector educativo

uma pesquisa de literatura é feita através de diferentes mecanismos de pesquisa na Internet, as palavras-chave são estabelecidas e diferentes estratégias de busca são criadas. Os documentos relevantes são selecionados e determinados conteúdos são analisados, extraídos e sintetizados. Os aspectos do geral para o particular são organizados e as conclusões necessárias são alcançadas através da indução - dedução.

Palavras chave: cultura organizacional; serviço ao cliente; instituições públicas; setor educacional.

\section{Introducción}

A medida que transcurre el siglo XXI, varias tendencias están causando un gran impacto en la cultura organizacional. Las instituciones tanto públicas, como privadas no pueden alejarse de esas realidades.

Los cambios están conformando un nuevo tipo de cultura organizacional, caracterizada por una nueva manera de pensar, de visualizar la organización, de desarrollar las actividades, y una actitud abierta hacia la innovación y la creatividad. Todo esto repercute en la efectividad general de la organización.

Cuando se trata de cambios organizacionales es conveniente entender que ellos deben sucederse como consecuencia de una actitud existente en la organización, y que los mismos deben ser congruentes con la cultura organizacional existente. Esto explica el fracaso de muchos programas de mejoramiento, cuando éstos son impuestos sin que hayan ocurridos los ajustes organizacionales requeridos para garantizar su éxito.

Las instituciones públicas del sector educativo están comprometidas con el éxito es por ello que deben contar con una cultura organizacional enfocada hacia el cliente.

Este trabajo tiene como objetivo abordar desde una perspectiva teórica la cultura organizacional enfocada al cliente en las instituciones públicas del sector educacional.

Para ello se hace una búsqueda de bibliografía mediante diferentes buscadores de Internet, se establecen las palabras claves y se construyen disímiles estrategias de búsqueda. Se seleccionan los documentos pertinentes y se analizan, se extraen y sintetizan determinados contenidos. Se organizan los aspectos de lo general a lo particular y se hacen necesarias inducciones y deducciones. 


\section{Desarrollo}

La cultura organizacional (CO) es el marco de referencia de los integrantes de las instituciones y pauta las conductas de las personas en ésta. En ese mismo sentido (Schein, 2010), la define como un patrón de supuestos básicos compartidos por un grupo, con el fin de resolver los problemas de adaptación externa e integración interna, y que han funcionado lo suficientemente bien como para ser considerados válidos y por lo tanto ser enseñados a nuevos miembros como la manera correcta de percibir, pensar y sentir en relación a esos problemas.

La cultura organizacional es la que moldea los comportamientos y actitudes en una empresa, por lo tanto, si el dar mejor servicio al cliente no está dentro del ADN de esa cultura es muy difícil que exista un esfuerzo coordinado que permita cumplir los objetivos y expectativas del cliente, y por ende, un servicio de excelencia. (Vidal Berrío M, 2015)

Montoya y Boyero (2013), expresan que el servicio al cliente es un conjunto de vivencias resultado del contacto entre la organización y el cliente, por lo que se considera la mejor manera de generar una relación adecuada, de la cual dependen su supervivencia y éxito. Según estos autores (Montoya, y otros, 2013), de la satisfacción que se brinde a través del servicio deriva que la organización conserve el cliente y, por eso, debe entender la importancia esencial de esta práctica.

En tiempos antiguos hablar de servicio se asociaba con labores menores e indignas, sin embargo, después de la década de los cincuenta y especialmente durante los setenta, se posicionó la idea afortunada de que el servicio al cliente era definitivamente no sólo un valor agregado sino también el factor que podía marcar la diferencia en relación al producto o servicio que se entregaba al cliente final.

En la literatura se enfoca este tema del cliente más hacia el externo, sin embargo los clientes internos son importantes también para el logro de una cultura organizacional que conlleve al éxito.

"Valorar al cliente interno nos ayuda a diseñar acciones más precisas sobre lo que nuestra empresa quiere ser en el futuro. Nos permite planificar con criterio estratégico y formular planes de trabajo acertados en función del logro de la visión. Es muy difícil tratar bien a los clientes externos, si primero no tratamos bien a los internos (...)”. (Jiménez, s/f) 
Cultura organizacional de servicio al cliente en las entidades públicas del sector educativo

El servicio al cliente, como cultura empresarial, aborda entonces varias dimensiones complementarias que establecen una balanza sobre la cual cada uno de sus componentes tiene el mismo peso, "en este sentido la libreta de calificaciones del cliente tiene cinco grandes asignaturas sobre las cuales la empresa debe invertir sus mejores esfuerzos con el fin de ofrecerle al cliente una verdadera experiencia que vaya más allá de sus expectativas. Estos elementos tienen que ver con:

1. El producto: Se refiere específicamente a lo que cada una de las empresas vende, es su producto tangible o intangible que recibe el cliente y sobre el cual emite un juicio acorde a la calidad, oportunidad, cantidad e incluso el valor entregado por él. Una mala calificación sobre el producto afecta la percepción de servicio ofrecido al cliente y aunque se tengan mecanismos de reposición y compensación para el cliente es posible que se afecte incluso el nivel de fidelización que se haya alcanzado.

2. Los procedimientos: Hace referencia a los mecanismos que la empresa ha institucionalizado para establecer los pedidos, la entrega del producto, los pagos y demás procedimientos que le permiten al cliente comunicarse y resolver las inquietudes que le surgen. Cuando los procedimientos son complejos o implican molestia para el cliente la calificación baja por cuanto se prefieren aquellas compañías que tienen un modelo de atención ágil, respetuoso y oportuno.

3. Las instalaciones: Es el sitio donde se presta el servicio y en este punto es importante considerar diversos aspectos que van a impactar directamente en los momentos de verdad a los cuales se somete la empresa en la prestación de su servicio, en este sentido podemos mencionar las vías de acceso, facilidad de parqueo, amplitud de las instalaciones, iluminación, ubicación de los productos, visibilidad de la información y de la imagen corporativa, etc. Una mala calificación en este punto hará que la percepción del servicio decaiga en detrimento no solamente del producto sino también de la marca y este es un riesgo que ninguna empresa está dispuesta a correr.

4. Tecnología disponible: La importancia de contar con sistemas de información robustos para el conocimiento de los clientes es fundamental, pero su funcionalidad deberá reflejarse en la capacidad que tenga el cliente para hacer uso de estos recursos, en este sentido se tiene en cuenta la posibilidad que tienen los clientes de hacer sus pedidos por Internet o bien por sistemas cerrados de comunicación, sistemas que le permitan a los clientes consultar en línea el estado de sus pedidos y facturación e igualmente que puedan tener acceso a las estadísticas de sus compras con la empresa, etc. La 
imposibilidad de contar con herramientas tecnológicas que permitan agilizar los procesos de comunicación y relación con la empresa se convertirá en un talón de Aquiles por cuanto el cliente estará atento a nuevas ofertas en el mercado que le permitan agilizar sus compras y sentir un modelo de atención personalizado entre otras cosas.

5. Información: Uno de los temas complejos de resolver en el diseño de una estrategia corporativa de servicio al cliente que tiene que ver precisamente con la identificación de los componentes de un sistema de información adecuado y ajustado a la expectativa del cliente, de tal manera que cada tipo de negocio deberá encontrar si la comunicación con el cliente es masiva o personalizada y si los esfuerzos y recursos que se dedican a este componente tienen retribución. El modelo de comunicación debe analizarse no solamente en la manera como se hace presente la empresa en el cliente y en su negocio si lo tiene, sino también en la calidad de la información que recibe cuando se comunica con la empresa y en este sentido vale la pena considerar hasta los mínimos detalles, como por ejemplo la oportunidad en la atención telefónica. Una información inadecuada, poco clara, confusa, incompleta o carente de veracidad traerá consecuencias en la decisión de compra del cliente e interpretará estas situaciones como descuido de la empresa frente al cliente y quizá juzgará con dureza las situaciones en las cuales sienta que no ha sido tomado en cuenta ni valorado en sus necesidades yexpectativas.

6. El prestador del servicio: Es la persona que atiende al cliente en el contacto real que tiene la empresa a través de sus productos o servicios, dependiendo el tipo de negocio el prestador del servicio tendrá la oportunidad no solamente de ofrecer al cliente una experiencia única frente a la empresa y su producto sino también la posibilidad de crear un proceso relacional con el cliente que va más allá de lo puramente transaccional, en este sentido el prestador del servicio no será entonces únicamente la persona que vende o atiende sino todo aquel que por su función tenga algún contacto directo o indirecto con el cliente, este concepto se refiere a que todos los integrantes de la organización tienen una responsabilidad con el cliente, no solamente con el producto que se entrega sino también con el esfuerzo permanente para que la calificación que ofrece el cliente a cada uno de los 5 puntos mencionados sea satisfactorio. El prestador del servicio entonces hace parte de una cultura organizacional que pasa por el concepto que tiene del cliente, de la importancia que le expresa, de la manera como establece su relación de servicio y de todos aquellos componentes que tienen que ver con los atributos propios del servicio al cliente, en este sentido no basta con tener buena actitud y disposición para atender y superar los requerimientos del cliente, es preciso que tenga autonomía y 
capacidad de decisión frente a todos aquellos aspectos recurrentes en la prestación del servicio". (Vecino, 2007)

Es evidente entonces que, se deben tener en cuenta los elementos más significativos del servicio al cliente y conscientes de que es necesario en su uso, el adquirir un propósito para el modelo se ve que, a medida que los negocios en general y el sector de servicios en particular se vuelven más competitivos; se agudiza la necesidad de una diferenciación competitiva significativa. Por ello, el propósito propio del servicio al cliente, cuya diferenciación se aboca en la determinación de actividades dirigidas a la satisfacción del cliente. Cada vez más esta diferenciación incluye una búsqueda de un desempeño superior, no sólo del servicio fundamental, sino de los elementos complementarios del servicio. El logro de esto requiere la formalización y la integración de las actividades del servicio al cliente en una función profesionalmente administrada, cuyo propósito, no sólo conducirá a satisfacer las necesidades de los clientes, sino más bien a incrementar la rentabilidad y prestigio de la empresa. (Lamb, 2004)

Fernández (2006) expresa que, para contribuir a la satisfacción del cliente en las instituciones educativas, se debe tomar en cuenta el compromiso de los directivos pues este es uno de los factores más importante, que a su vez "denota una gran confianza externa de cara a hacer una valoración positiva de dicho centro, además de hacer que el funcionamiento del centro sea el más adecuado". Sin embargo, para que ese compromiso se logre y llegar a ser una institución eficaz y de calidad es necesario comenzar a difundir los valores, normas, objetivos de la institución, aunque muchas veces se torne complicado.

La cultura de una institución influye de manera decisiva en sus política y, lógicamente, en sus técnicas y procedimientos. (Puchol, 2007)

Cabe agregar que la cultura organizacional es importante en el desarrollo de la eficiencia, eficacia para alcanzar objetivos, pero cuando las políticas están implantadas en base al entorno y a los intereses de los empleados y la sociedad permiten tomar decisiones, y brindar una atención de calidad en los entes públicos; porque la motivación es parte fundamental de tal manera que estos deben desarrollar sus habilidades, técnicas, y de comunicación para brindar un mejor servicio al cliente. 
En este propósito, muchas investigaciones sobre el contexto organizacional han señalado que es más probable obtener mejores resultados laborales, un alto desempeño, compromiso o cooperación cuando las personas se sienten parte de un grupo de trabajo con un clima organizacional satisfactorio, cuando perciben que tienen las competencias adecuadas para desarrollar su trabajo y cuando son recompensados y reconocidos como agentes significativos de los resultados organizacionales. (Chiang, 2010)

La administración de instituciones, particularmente de la gestión organizacional, el talento humano no puede dejar de estar incluido en la agenda de toda organización moderna. Más allá del tipo de empresa y negocio, la gente siempre es una de las dimensiones que impactan en la estrategia y en los resultados. La organización es su gente, y ésta cumple un rol clave cualquiera sea la situación del entorno (Gadón, 2010). En la actualidad los recursos humanos son los que hacen la diferencia real para lograr las metas y logros organizacionales. De hecho, una ventaja en particular de una organización depende en forma creciente del activo más valioso de una empresa, sus empleados y trabajadores.

De manera general, una institución de educación superior debe contar con una cultura organizacional enfocada tanto a los clientes internos y como externos.

\section{- Clientes internos:}

\section{Personal docente y de investigación}

"Es el motor que genera y potencia el proceso de enseñanza-aprendizaje, por lo cual requiere de una atención amable, oportuna y sensible a sus necesidades de capacitación, desarrollo profesional, actualización de conocimientos pedagógicos y tecnológicos, entre otros. Por lo tanto, el personal directivo y administrativo deberá realizar todos los esfuerzos para crear y conservar relaciones laborales adecuadas tendientes a la integración de los docentes en la institución.” (Ojeda Zambrano, 2011)

\section{Personal directivo}

"El personal directivo constituye el puntal que lidera la gestión de las instituciones de educación superior, en base a la planificación estratégica que deberá tener como punto focal al conglomerado 
estudiantil y demás organizaciones y sociedad a la que sirve. El personal directivo requiere información precisa, oportuna y constantemente actualizada, pues de ello depende el adecuado proceso de toma de decisiones. Por lo tanto, los proveedores internos, en este caso, deben proporcionarles toda la información y recursos necesarios con un trato amable y asertivo." (Ojeda Zambrano, 2011)

\section{Personal administrativo y de servicios}

“El personal administrativo, en todos sus niveles y responsabilidades, tiene a su cargo el desarrollo de procesos que apoyan la gestión operativa de la institución; por lo tanto, existe un gran número de funcionarios que son, tanto proveedores de otros funcionarios, como clientes internos de otros. La línea base de servicio al cliente se hace indispensable también para este tipo de cliente, puesto que de igual manera el personal administrativo merece ser tratado con amabilidad, oportunidad y proactividad. Es indispensable reflexionar aquí, que en el caso de existir incumplimiento por parte los proveedores internos, los procesos se retrasan y perjudican así el flujo de los siguientes procesos a cargo de otros clientes internos, lo cual influye negativamente hacia los productos finales destinados a los clientes externos. Ejemplos de ello pueden ser: trámites académicos de matriculación y de graduación que no son completados en las fechas previstas o en las condiciones establecidas para el efecto.” (Ojeda Zambrano, 2011)

\section{- Clientes externos}

\section{Estudiantes}

“(...) es imprescindible realizar investigaciones sobre las necesidades y requerimientos del estudiante, considerando integralmente su proyección profesional, laboral y humana (...) El personal docente quien lo atiende directamente debe hacerlo no solamente con la tan valorada vocación, sino también con la visión de ofrecerle las posibilidades de crecimiento intelectual y personal y el desarrollo de sus competencias. La consideración en el trato, el respeto de su individualidad, el acercamiento y la empatía respecto al alumno son esenciales, así como la obligación de proveerle un producto de alta calidad, es decir, el conocimiento actualizado. El personal directivo y administrativo tiene, por su parte, el deber de facilitar al estudiante todo tipo de trámites y también realizar un seguimiento académico y de ser posible, proveerle el acercamiento al ámbito laboral cuando el alumno ya ha 
cumplido con determinados requisitos básicos para iniciar su ingreso a la esfera de su futura profesión”. (Ojeda Zambrano, 2011)

\section{Instituciones gubernamentales y privadas}

Las instituciones de educación superior tienen la responsabilidad de crear y fortalecer relaciones con diferentes instituciones gubernamentales y privadas que favorezcan el desarrollo administrativo, académico y de investigación, a través de convenios institucionales, acuerdos de cooperación, entre otras figuras. En tal razón, los clientes de este grupo serán básicamente otras instituciones de educación superior, instituciones de educación media, ministerios, gobiernos seccionales, secretarías gubernamentales, empresas industriales y de servicios, entre otros. El trato para estos clientes deberá igualmente fundamentarse en el respeto y buscará fortalecer las relaciones que se desarrollan a través de sus directivos y funcionarios. Se les deberá ofrecer información completa y oportuna, de tal manera que los distintos acuerdos y trámites se realicen bajo los parámetros técnicos especificados.

\section{Consideraciones finales}

La cultura organizacional enfocada al cliente en las instituciones públicas del sector educacional debe caracterizarse por la satisfacción del estudiante que es la razón de ser de toda institución educativa, por procederes simples, por hacer de las instalaciones centros confortables, por tener un flujo y canales de comunicación adecuados y por contar con un personal responsable.

La cultura organizacional enfocada al cliente en las instituciones públicas de educación exigen contar con profesores preparados, actualizados científicamente, que utilicen adecuadamente las tecnologías y los modelos pedagógicos modernos, que se retroalimenten del proceso académico; con directivos capaces de integrar equipos y establecer relaciones con terceros.

Para desarrollar una excelente cultura organizacional enfocada en el servicio al cliente en las instituciones públicas del sector educacional es imperativo que exista un placentero ambiente laboral, con el trabajar en valores y se divulguen adecuadamente las normas y objetivos de la institución. Donde se motive a los trabajadores en su realización personal y profesional, exista el diálogo, se favorezca la innovación y la creatividad, se premie la responsabilidad y la productividad. 
Crear una cultura de servicio al cliente en las instituciones públicas educativas implica una estrategia organizacional donde se incluyan todos los clientes, dónde a partir de una evaluación diagnóstica se implementen acciones para el mejoramiento de la satisfacción, fidelidad y compromiso.

\section{Referencias Bibliográficas}

Chiang, M. 2010. Relaciones Entre el Clima Organizacional y la Satisfacción Laboral. España: Servicios Editoriales, 2010.

Gadón, F. 2010. La Gestión del Talento en Tiempos de Cambio. Argentina: Ediciones Granica S.A., 2010.

Jimenez, J.C. s/f. Una cultura organizacional orientada al cliente interno. [En línea] s/f. [Citado el: 02 de febrero de 2017.] http://www.grandespymes.com.ar/2012/04/01/una-cultura-organizacionalorientada-al-cliente-interno/.

Lamb. 2004. Servicio al cliente. 2004.

Montoya, C. y Boyero, M. 2013. El CRM como herramienta para el servicio al cliente en la organización. Visión de futuro 17(1). 2013.

Ojeda Zambrano, C. 2011. Calidad del servicio al cliente en instituciones de educación superior. [En línea] 2011. [Citado el: 12 de abril de 2017.] https://www.gestiopolis.com/calidad-del-servicio-alcliente-en-instituciones-educacion-superior/.

Puchol, L. 2007. Dirección y Gestión de Recursos Humano. España: Ediciones Díaz de Santos, 2007. Schein, E. 2010. Organizational Culture and leadership. United States of America: John Wiley\& Son, Inc., 2010.

Vecino, J.M. 2007. La cultura del servicio al cliente como estrategia gerencial. [En línea] 2007. [Citado el: 20 de abril de 2017.] http://www.dinero.com/columna-del-lector/opinion/articulo/lacultura-del-servicio-cliente-como-estrategia-gerencial/50520. 
Cultura organizacional de servicio al cliente en las entidades públicas del sector educativo

Vidal Berrío M, L. 2015. Cultura organizacional puede mejorar atención al cliente. [En línea] 2015. [Citado el: 02 de abril de 2017.] https://elcapitalfinanciero.com/cultura-organizacional-puedemejorar-atencion-al-cliente/. 